\title{
Diagnostic Performance of Line Probe Assay for the Diagnosis of Rifampicin and Isoniazid Resistant Tuberculosis in a Resource- Poor Country
}

Laura Madukaji $^{1}$; Francis Enenche Ejeh ${ }^{2}$; Eke Ofuche ${ }^{1}$; Jay Osi Samuel ${ }^{1}$; Femi Owolagba $^{1}$; Ughweroghene Omo-Emmanuel $^{3}$, Prosper Okonkwo ${ }^{1}$

${ }^{1}$ APIN, Public Health Initiative, Abuja, Nigeria .

${ }^{2}$ Department of Veterinary Microbiology, University of Maiduguri, Nigeria..

${ }^{3}$ USAID, Abuja, Nigeria.

Corresponding Author Francis Enenche Ejeh

Mobile:

$+2348057243878$

E mail:

enenchefrancis@yahoo .com;

enenchefrancis@unim aid.edu.ng

Key words: sensitivity; specificity; multidrug-resistant tuberculosis; Nigeria; Mycobacterium tuberculosis complex; microbial resistance
Background and study aim: The use of LPA is still new in Nigeria and only available in TB reference laboratories. In this study, the performance of LPA version 2.0 was evaluated for the detection of resistant to first-line anti-TB drugs.

Patients and Methods: We evaluated the performance of LPA version 2.0 for the detection of rifampicin (RIF) and isoniazid (INH) resistance. Sputum samples from 223 participants were subjected to phenotypic drug susceptibility testing (PDST) and LPA. Statistical analyses included calculation of sensitivity, specificity, positive and negative predictive values. Cross tabulation was done along the kappa test to measure the degree of agreement between PDST and LPA. P-Value > 0.05 was considered significant.
Results: The overall sensitivity and specificity of $89.6 \% \quad(95 \%$ C.I 82.5 $94.5 \%$ ) and $65.4 \%$ (95\% C.I $44.3-82.7 \%$ ) for detection of RIF resistance; for INH they were 76.6 (95\% C.I $67.5-84.5 \%)$ and $76.7 \%$ (95\% C.I $49.5-82.6 \%)$; and for MDR-TB, they were $67.0 \%(95 \%$ C.I $56.4-76.5 \%)$ and $72.0 \%$ (95\% C.I 57.6$83.7 \%)$. The kappa values were 0.53 (0.001), $0.38(\mathrm{p}=0.000)$ and $0.36(\mathrm{p}=$ 0.000 ) for the detection of RIF, INH and MDR-TB. There was moderate agreement between PDST and LPA for detection of RIF $(\kappa=0.57 ; \mathrm{P}=0.0001)$, INH $(\kappa=$ $0.44 ; \mathrm{P}=0.0001)$, MDR-TB $(\kappa=0.43 ; \mathrm{P}$ $=0.001)$.

Conclusion: The Line probe assay has good sensitivity and specificity for detecting rifampicin and isoniazid. However, the overall performance is moderate; this should be considered when interpreting the assay's results.

\section{INTRODUCTION}

Tuberculosis is a significant public health challenge. The world health organization (WHO) reported that over 10.4 million cases and 1.7 million deaths occur globally on an annual basis due to tuberculosis [1]. Nigeria and five other countries account for $60 \%$ of the global tuberculosis burden [2]. The incidence of tuberculosis cases in Nigeria was estimated at $345000-890000$, with a potential of zoonotic tuberculosis grossly under-reported [3]. The EndTB program's significant challenges include rapid and accurate diagnosis of tuberculosis, development of drug resistance, and timely detection of drug-resistant tuberculosis. Other challenges include poverty and HIV [4].

The diagnosis of tuberculosis in Nigeria is made mainly by acid-fast microscopy and culture and chest $\mathrm{X}$ ray. These methods of tuberculosis diagnosis are faced with numerous problems. Acid-fast has the following challenges: low turnaround time but the technique has reduced sensitivity and low specificity; it is unable to differentiate between non-tuberculous mycobacteria (NTM) and Mycobacterium tuberculosis complex (MTBC) [5, 6]. Culture is the gold standard for the diagnosis of tuberculosis. However, the method requires 1-12 weeks before results are available. Also, contamination limits the use of culture for the diagnosis of 
tuberculosis. The utilization of a chest X-ray (CXR) is limited because of radiation exposure, and the result is often subjective [7]. It is not suitable for TB diagnosis as it is known that CXR is an essential tool for early detection of tuberculosis (TB), CXR has high sensitivity, but limited specificity for detecting pulmonary TB as many CXR abnormalities that are consistent with pulmonary TB are also seen in several other lung pathologies [8].

The conventional method for diagnosing drugresistant tuberculosis was based on phenotypic drug susceptibility testing (PDST), agar or brothbased media. This method, as with culture, is time-consuming and prone to contamination. The World Health Organization endorsed using a line probe assay for diagnosing tuberculosis and detecting resistance to rifampicin and isoniazid [9].

Line Probe Assay in diagnosing tuberculosis is still new in Nigeria and only available in reference laboratories. This study evaluates the performance of the line probe assay version 2.0 to detect rifampicin and isoniazid by using conventional $1 \%$ phenotypic drug susceptibility testing as the reference standard in Nigeria.

\section{METHODS}

\section{Study participant:}

This study was a prospective analysis of hypothetical Drug-Resistant TB cases screened by acid-fast bacilli (AFB) test. Study participants ranged from 15 to 80 years who were enrolled in DR-TB treatment and care. At the time of screening for TB, the HIV status of participants were documented by the DOT focal person. Sputum samples (clinical specimens) were collected from participants who gave their consent. These participants were recruited from DOT centres in Benue, Nasarawa, FCT, Niger and Kogi States. FCT being the federal capital territory, has more and well-structured DOT. Hence, we had more participants from FCT. We recruited 223 patients from the Directly Observed Treatment Short-Course (DOT) centres in 5 states in the North Central zone of Nigeria. One sputum sample was collected from each patient in $50-\mathrm{mL}$ wide-mouthed sterile Falcon tubes and processed in Biosafety cabinet type II. Written informed consent was obtained from all patients at the time of enrolment into the study from October 2018 to August 2019.

\section{Inclusion criteria:}

- Availability of test results for LPA and PDST

- All ages

- Patients with HIV results

- Ability to produce sputum without an inducement

\section{Exclusion criteria:}

- Extra-pulmonary tuberculosis

- Invalid LPA results

- Inconclusive results

- Contamination of PDST culture

- Non-availability of corresponding results for both LPA and PDST

\section{Study setting:}

Every state in Nigeria has a Directly Observed Treatment Short-course (DOT) centre. However, there is still inadequate laboratory testing, as only nine culture laboratories are functional. To increase TB culture and LPA, the National TB and Leprosy Control Program (NTBLCP) strengthened the Specimen referral system through a national mapping of the states and zones and linking them to their respective testing sites (reference laboratories). Both old and newly enrolled patients on treatment are enlightened about TB and anti-TB therapy, including drug refill frequency and clinic days. Clinic days are for health talk and general drug pick-up, and this is when the DS-TB \& DR-TB focal persons move to their assigned DOT centres to collect specimens for the first culture/LPA test $\&$ follow up. Specimens are collected, triple packaged and transported to the linked reference laboratories. Test results are conveyed to the DOT centres through the same DS-TB and DR-TB focal person. Samples were processed at the Zankli TB Reference Laboratory. Zankli TB reference Laboratory is one of the reference laboratories for the Northern States in Nigeria. The reference laboratory has the capacity of running AFB microscopy, Genexpert, first and second-line phenotypic culture and DST, first and secondline genotypic Line Probe Assay (LPA). This study's samples were processed using LPA Genotype MTBDRplus, and culture-Drug Susceptibility Testing Lowenstein Jensen proportion method (LJ-DST).

\section{Culture:}

We decontaminated and digested the sputum samples by the NALC-NaOH method (NALC $2.9 \% \& \mathrm{NaOH} 4 \%$ ). Afterwards, the mixture was 
centrifuged at $3000 \mathrm{~g}$ for 15 minutes. In each tube, the pellet was suspended in $2.5 \mathrm{ml}$ of phosphate buffer $\mathrm{pH} 6.8$, inoculated onto sterile Lowenstein Jensen agar slants and then incubated. We stored the remaining concentrates (pellet and buffer) at $4^{\circ} \mathrm{C}$ for future use. The slants were checked in the first week for contamination and or NTM to enable a prompt repeat of the culture process. On the eight weeks, cultures were read according to the National TB Standard Procedure.

\section{Phenotypic Drug Susceptibility Testing (PDST):}

We used conventional $1 \%$ proportion phenotypic drug susceptibility testing (DST) on Lowenstein Jensen (LJ) medium. The first-line drug's final concentration was isoniazid $(0.2 \mathrm{ug} / \mathrm{ml})$ and rifampin $(40 \mathrm{ug} / \mathrm{ml})$. The cultures with confirmed MTB were sub-cultured onto the drug-containing sterile LJ slants and non-drug containing slants (controls) and incubated for four weeks. Cultures were read after four weeks of incubation. All procedures were carried out according to the National Standard operating procedure (SOP) for LJ Culture/DST

\section{Line Probe Assay Version 2.0 (LPA):}

A chemical DNA extraction method (Genolyse kit from Hain Lifescience, Germany) was used to extract DNA from the concentrate. The manufacturer's instruction was strictly followed to get the DNA extracts. Polymerase chain reaction (PCR) was performed using $35 \mu \mathrm{l}$ of primer nucleotide mix, $10 \mu \mathrm{l}$ of Taq DNA polymerase-PCR buffer mix and $5 \mu 1$ of supernatant in a final volume of $50 \mu 1$. Amplification was done in a thermal cycler. Twincubator was used to perform Reverse hybridization. Genotype MTBDRplus kit instruction was strictly followed to find any deletion in wild-type gene loci and mutations in rpoB (RNA polymerase $B$ subunit), Kat G (catalase-peroxidase) and inhA (inoyl coenzyme A reductase) loci.

The hybridization strips consist of 27 reaction zones (6 control probes and 21 probes for mutation). All control probes must be present in a valid test, and the absence and the presence of any mutation band imply resistance to the particular antibiotic tested. The control probes include conjugate control, amplification control, Mycobacterium tuberculosis complex control (TUB), rpoB amplification control, inhA amplification control and katG amplification control. For detecting rifampicin resistance, the rpoB gene (coding for the $\beta$-subunit of the RNA polymerase) and high-level. For INH resistance, the katG gene (coding for the catalaseperoxidase) is examined. The promoter region of the inhA gene (coding for the NADH enoyl ACP reductase) was examined to detect low-level INH resistance.

LPA was compared with the gold standard (phenotypic culture/DST) to evaluate the sensitivity, specificity, positive predictive value (PPV), and negative predictive value (NPV). All the results for the samples were analyzed to compare the diagnostic performance of the assays used. We used Graph pad prism version 3.5 to perform the statistical analyses.

\section{RESULTS}

\section{Demographic characteristic of study participants:}

A total of 223 suspected Patients with TB were recruited in the study. Of the 223 recruited in the study, 141 patients' specimens were included in the study analyses, while $82(36.8 \%)$ were excluded from the study analyses based on the conditions described in figure 1. Among all the patients' specimens, $168(72.1 \%)$ were culture positive, of which $27(12.1 \%)$ were identified as NTM. Thirty-four (15.3\%) were negative by culture on LJ, and 21 (9.4\%) were contaminants. LPA had 14 (6.0\%) invalid results, 68 (30.5\%) specimens with valid LPA results were excluded from the study analyses because they do not have the corresponding result for phenotypic drug susceptibility testing on LJ slant. Other samples that were excluded from the study analyses are presented in figure 1.

The mean age of those included in the study was $39.2 \pm 15.2$ years. Eighty-one $(57.9 \%)$ were males, and $60(42.7 \%)$ were females. Patients with new TB cases were 90 (70.2\%), and retreatment cases were $42(29.8 \%)$. Thirty-one $(22.0 \%)$ were HIV positive $63(44.4 \%)$ were from FCT (Table 1).

\section{Diagnostic performance of LPA Using Phenotypic Drug Susceptibility Testing (Lowenstein-Jensen) as Gold Standard:}

To assess the performance of LPA test in detecting RMP, INH, and MDR-TB, culture LJ drug susceptibility testing was used as the gold 
standard. LPA reported sensitivity, specificity, positive and negative predictive values of $89.6 \%$ (95\% C.I: 82.5 - 94.5\%), 65.4 (95\% C.I: $44.3-$ $82.3 \%), 92.0 \%$ (95\% C.I: $85.3-96.3 \%$ ) and $58.6 \%$ (95\% C.I: 38.9 - 76.5\%) in detecting RMP. There was a moderate agreement $(\mathrm{kappa}=$ 0.53; $\mathrm{P}=0.000$ ) between LPA and culture LJ DST in detecting rifampicin resistant TB. The sensitivity and specificity of LPA in detecting INH resistance were $76.6 \%$ (95\% C.I: $67.5-$ $84.3 \%$ ) and $67.7 \%$ (95\% C.I: $49.5-82.6 \%$ ). There was a moderate agreement $($ Kappa $=0.38$; $\mathrm{P}=0.000$ ) between LPA and culture in detecting INH resistant TB. Also, LPA and culture had moderate agreement $($ Kappa $=0.36 ; \mathrm{P}=0.000)$ in detecting MDR-TB. LPA reported a sensitivity and specificity of 67.0 (95\% C.I: 56.4 $-76.5 \%$ ) and $72 \%$ (95\% C.I: $57.6-83.7 \%$ ) for detecting MDR-TB (table 2).

LPA reported sensitivity and specificity of 83.3\% (95\% C.I:65.3-94.4\%) and $75.0 \%$ (95\% C.I: $19.4-99.4 \%$ ) respectively in detecting rifampicin resistant TB among Patients with HIV. The agreement between both tests for detection of RMP-resistant TB was moderate (Kappa $=0.40 ; \mathrm{P}=0.016)$, the sensitivity and specificity of LPA to detect isoniazid-resistant TB among patients with HIV were 73.9 (95\% C.I: $51.6-89.8 \%)$ and 50.0 (15.7 - 84.3\%). Also, LPA reported sensitivity and specificity of
$57.1 \%$ (95\% C.I: $34.1-78.2 \%$ ) and $60.0 \%$ (95\% C.I: $26.3-87.8 \%$ ) to detect INH resistant TB among HIV patient. The agreement between both tests for the detection of INH resistance among Patients with HIV was fair (Kappa $=0.22$; $\mathrm{P}=$ 0.213). Although, LPA performance in detecting INH and MDR -TB were fair compared with culture, the results for HIV negative patient indicated better performance by LPA (Table 3 and 4). The sensitivity and specificity of LPA to detect rifampicin resistant TB among HIV negative patient were $92.1 \%$ (95\% C.I: 84.3 $96.7 \%$ ) and $63.6 \%$ (95\% C.I: $40.7-82.2 \%)$. There was moderate agreement (Kappa $=0.57 ; \mathrm{P}$ $=0.000$ ) between LPA and culture to detect RMP resistant TB among HIV negative patients. The sensitivity, positive and negative predictive values of LPA to detect INH-resistant TB among HIV-infected patients was $77.4 \%$ (95\% C.I: 66.9 - $85.8 \%$ ), $73.1 \%$ (95\% C.I: 52.2 - 88.4\%), $90.3 \%$ (95\% C.I: $81.0-96.0 \%)$ and $50.0 \%$ (95\%C.I: 33.4 - 66.7\%) respectively. The sensitivity, positive and negative predictive values of LPA to detect MDR TB among HIV negative patients were $70.0 \%$ (95\% C.I: 57.9 - 80.4\%), $75.0 \%$ (95\% C.I: 58.8 - 87.3\%), $83.1 \%$ (95\% C.I: $71.0-$ 91.6\%) and $58.9 \%$ (95\%C.I: $44.2-72.4 \%$ ); the Kappa agreement was 0.43 . (Table 4).

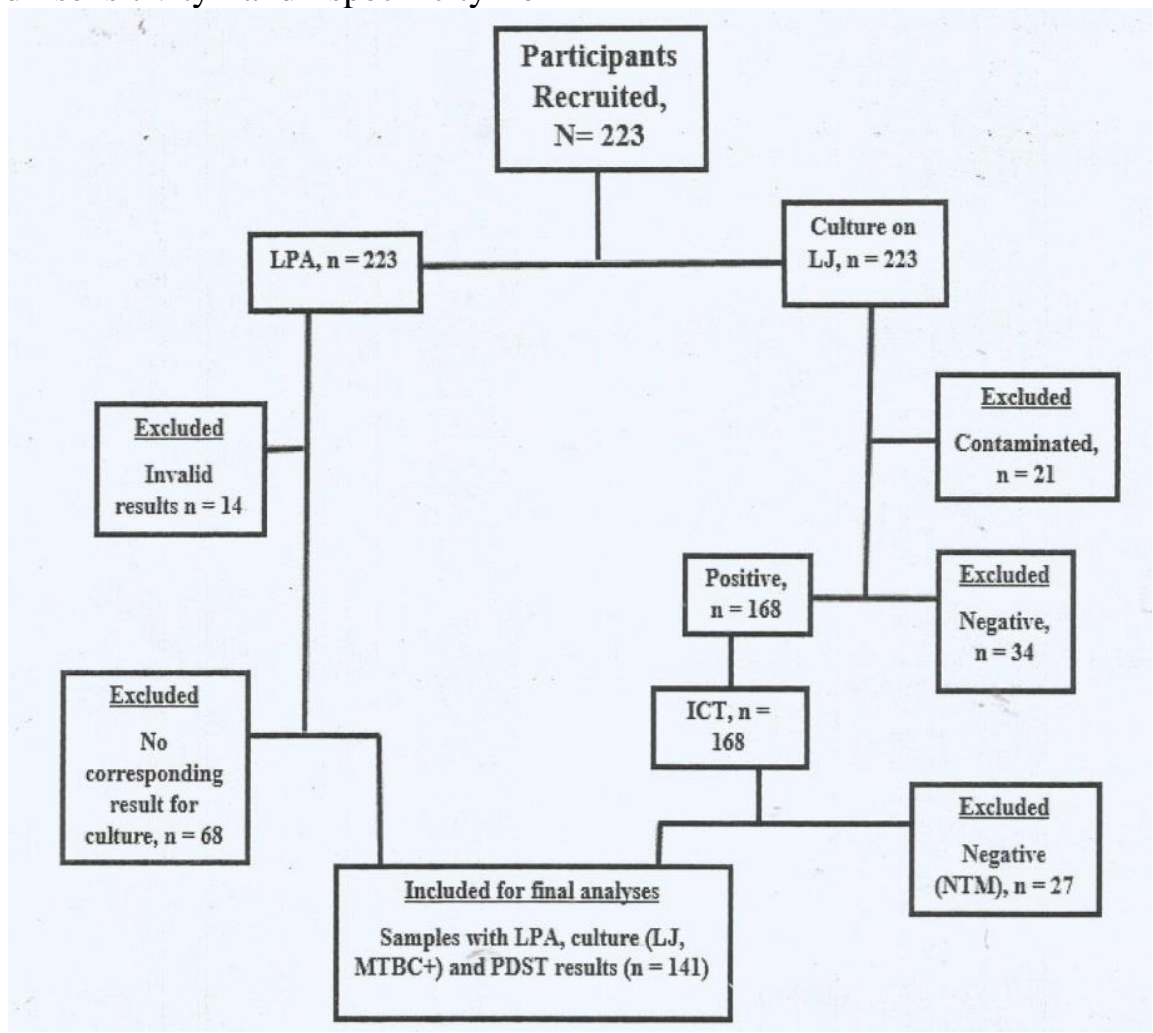

Figure (1): Schematic Representation of the Workflow. 
Table (1): Demographic and Clinical Characteristics of Study Participants.

\begin{tabular}{|l|c|c|}
\hline Parameters & Frequency & Percentages (\%) \\
\hline Age (Mean \pm SD) & $39.17 \pm 15.4$ & \\
\hline Sex & & 57.4 \\
\hline Male & 81 & 42.7 \\
\hline Female & 60 & 22.0 \\
\hline HIV Status & & 78.0 \\
\hline Positive & 31 & \\
\hline Negative & 110 & 7.1 \\
\hline State & & 44.4 \\
\hline Benue & 10 & 17.7 \\
\hline FCT & 63 & 19.9 \\
\hline Kogi & 25 & 10.6 \\
\hline Nasarawa & 28 & \\
\hline Niger & 15 & \\
\hline
\end{tabular}

HIV = Human Immunodeficiency Syndrome; FCT = Federal Capital Teritory.

Table (2): The overall Performance of LPA test as compared to LJ DST in detecting resistance to rifampicin, isoniazid, and MDR-TB.

\begin{tabular}{|c|c|c|c|c|c|c|c|}
\hline \multicolumn{8}{|c|}{$\begin{array}{l}\text { Gold Standard: Culture on Lowenstein- } \\
\text { Jensen Agar Slant }\end{array}$} \\
\hline \multicolumn{2}{|c|}{ LPA Assay } & \multicolumn{2}{|c|}{\begin{tabular}{|l|} 
Rifampicin \\
\end{tabular}} & \multicolumn{2}{|c|}{ Isoniazid } & \multicolumn{2}{|c|}{ MDR } \\
\hline & & Resistant & Susceptible & Resistant & Susceptible & Resistant & Susceptible \\
\hline & & $(\mathrm{n}=115)$ & $(\mathrm{n}=26)$ & $(\mathrm{n}=107)$ & $(\mathrm{n}=34)$ & $(n=67)$ & $(\mathrm{n}=18)$ \\
\hline LPA Assay & Resistant & 103 & 9 & 82 & 11 & 61 & 14 \\
\hline & Susceptible & 12 & 17 & 25 & 23 & 6 & 4 \\
\hline $\begin{array}{l}\text { Sensitivity } \\
\text { (95\% C.I) }\end{array}$ & & $\begin{array}{c}89.6 \\
(82.5-94.5) \\
\end{array}$ & & $\begin{array}{c}76.6 \\
(67.5-84.3) \\
\end{array}$ & & $\begin{array}{c}67.0 \\
(56.4-76.5) \\
\end{array}$ & \\
\hline $\begin{array}{l}\text { Specificity } \\
(95 \% \text { CI })\end{array}$ & & $\begin{array}{c}65.4 \\
(44.3-82.7) \\
\end{array}$ & & $\begin{array}{c}67.7 \\
(49.5-82.6) \\
\end{array}$ & & $\begin{array}{c}72.0 \\
(57.6-83.7) \\
\end{array}$ & \\
\hline $\begin{array}{l}\text { Positive } \\
\text { Predictive } \\
\text { Value } \quad(95 \% \\
\text { CI) }\end{array}$ & & $\begin{array}{c}92.0 \\
(85.3-96.3)\end{array}$ & & $\begin{array}{c}88.2 \\
(79.8-94.0)\end{array}$ & & $\begin{array}{c}81.3 \\
(70.6-89.4)\end{array}$ & \\
\hline $\begin{array}{l}\text { Negative } \\
\text { Predictive } \\
\text { Value } \quad(95 \% \\
\text { CI }) \\
\end{array}$ & & $\begin{array}{c}58.6 \\
(38.9-76.5)\end{array}$ & & $\begin{array}{c}47.9 \\
(33.3-62.8)\end{array}$ & & $\begin{array}{c}54.6 \\
(41.9-66.9)\end{array}$ & \\
\hline $\begin{array}{l}\text { Kappa }(P \\
\text { Value }) \mathbf{l} \\
\end{array}$ & & $0.53(0.000)$ & & $0.38(0.000)$ & & $0.36(0.000)$ & \\
\hline
\end{tabular}

LPA = Line Probe Assay; MDR = Multi-drug Resistance; C.I = Confidence Interval; MTBDR = Mycobacterium tuberculosis drug resistance, LJDST = Lowenstein-Jensen Drug Susceptibility Testing. 
Table (3): Performance of LPA test as compared to LJ DST in detecting resistance to rifampicin, isoniazid, and MDR-TB among HIV Patients.

\begin{tabular}{|c|c|c|c|c|c|c|c|}
\hline \multicolumn{8}{|c|}{$\begin{array}{l}\text { Gold Standard: Culture on Lowenstein- } \\
\text { Jensen Agar Slant }\end{array}$} \\
\hline \multicolumn{2}{|l|}{ LPA Assay } & \multicolumn{2}{|c|}{ Rifampicin } & \multicolumn{2}{|c|}{ Isoniazid } & \multicolumn{2}{|c|}{ MDR } \\
\hline & & Resistant & Susceptible & Resistant & Susceptible & Resistant & Susceptible \\
\hline & & $(n=30$ & $(n=4)$ & $(n=23)$ & $(n=8)$ & $(n=21)$ & $(n=10)$ \\
\hline MTBDR-PLUS & Resistant & 25 & 1 & 17 & 4 & 12 & 4 \\
\hline & Susceptible & 5 & 3 & 6 & 4 & 9 & 6 \\
\hline $\begin{array}{l}\text { Sensitivity }(95 \% \\
\text { C.I) }\end{array}$ & & $\begin{array}{c}83.3 \\
(65.3-94.4)\end{array}$ & & $\begin{array}{c}73.9 \\
(51.6-89.8)\end{array}$ & & $\begin{array}{c}57.1 \\
(34.1-78.2)\end{array}$ & \\
\hline $\begin{array}{l}\text { Specificity }(95 \% \\
\mathrm{CI})\end{array}$ & & $\begin{array}{c}75.0 \\
(19.4-99.4)\end{array}$ & & $\begin{array}{c}50.0 \\
(15.7-84.3)\end{array}$ & & $\begin{array}{c}60.0 \\
(26.3-87.8)\end{array}$ & \\
\hline $\begin{array}{l}\text { Positive } \\
\text { Predictive Value } \\
(95 \% \mathrm{CI})\end{array}$ & & $\begin{array}{c}96.2 \\
(80.4-100.0)\end{array}$ & & $\begin{array}{c}81.0 \\
(58.1-94.6)\end{array}$ & & $\begin{array}{c}75.0 \\
(47.7-92.7)\end{array}$ & \\
\hline $\begin{array}{l}\text { Negative } \\
\text { Predictive Value } \\
(95 \% \mathrm{CI})\end{array}$ & & $\begin{array}{c}37.5 \\
(8.5-75.6)\end{array}$ & & $\begin{array}{c}40.0 \\
(12.2-73.6)\end{array}$ & & $\begin{array}{c}40.0 \\
(16.3-67.8)\end{array}$ & \\
\hline $\begin{array}{l}\text { Kappa }(\mathbf{P} \\
\text { Value) }\end{array}$ & & $0.40(0.016)$ & & $0.22(0.213)$ & & $0.15(0.372)$ & \\
\hline
\end{tabular}

LPA = Line Probe Assay; MDR = Multi-drug Resistance; C.I = Confidence Interval; MTBDR = Mycobacterium tuberculosis drug resistance, LJDST = Lowenstein-Jensen Drug Susceptibility Testing

Table (4): Performance of LPA test as compared to LJ DST in detecting resistance to rifampicin, isoniazid, and MDR-TB among HIV Negative patients.

\begin{tabular}{|c|c|c|c|c|c|c|c|}
\hline \multicolumn{8}{|c|}{$\begin{array}{l}\text { Gold Standard: Culture on Lowenstein- } \\
\text { Jensen Agar Slant }\end{array}$} \\
\hline \multicolumn{2}{|l|}{ LPA Assay } & \multicolumn{2}{|c|}{ Rifampicin } & \multicolumn{2}{|c|}{ Isoniazid } & \multicolumn{2}{|c|}{ MDR } \\
\hline & & Resistant & Susceptible & Resistant & Susceptible & Resistant & Susceptible \\
\hline & & $(n=88)$ & $(n=22)$ & $(n=84)$ & $(n=26)$ & $(\mathrm{n}=70)$ & $(n=40)$ \\
\hline MTBDR-PLUS & Resistant & 81 & 8 & 65 & 7 & 49 & 10 \\
\hline & Susceptible & 7 & 14 & 19 & 19 & 21 & 30 \\
\hline $\begin{array}{l}\text { Sensitivity (95\% } \\
\text { C.I) }\end{array}$ & & $\begin{array}{c}92.1 \\
(84.3-96.7) \\
\end{array}$ & & $\begin{array}{c}77.4 \\
(66.9-85.8) \\
\end{array}$ & & $\begin{array}{c}70.0 \\
(57.9-80.4) \\
\end{array}$ & \\
\hline $\begin{array}{l}\text { Specificity }(95 \% \\
\text { CI) }\end{array}$ & & $\begin{array}{c}63.6 \\
(40.7-82.8) \\
\end{array}$ & & $\begin{array}{c}73.1 \\
(52.2-88.4) \\
\end{array}$ & & $\begin{array}{c}75.0 \\
(58.8-87.3) \\
\end{array}$ & \\
\hline $\begin{array}{l}\text { Positive } \\
\text { Predictive Value } \\
(95 \% \text { CI })\end{array}$ & & $\begin{array}{c}91.0 \\
(83.0-96.0)\end{array}$ & & $\begin{array}{c}90.3 \\
(81.0-96.0)\end{array}$ & & $\begin{array}{c}83.1 \\
(71.0-91.6)\end{array}$ & \\
\hline $\begin{array}{l}\text { Negative } \\
\text { Predictive Value } \\
(95 \% \text { CI }) \\
\end{array}$ & & $\begin{array}{c}66.7 \\
(43.0-85.4)\end{array}$ & & $\begin{array}{c}50.0 \\
(33.4-66.7)\end{array}$ & & $\begin{array}{c}58.9 \\
(44.2-72.4)\end{array}$ & \\
\hline $\begin{array}{l}\text { Kappa }(\mathbf{P} \\
\text { Value })\end{array}$ & & $0.57(0.000)$ & & $0.44(0.000)$ & & $0.43(0.000)$ & \\
\hline
\end{tabular}

LPA = Line Probe Assay; MDR = Multi-drug Resistance; C.I = Confidence Interval; MTBDR = Mycobacterium tuberculosis drug resistance.

\section{DISCUSSION}

This study's findings showed that the line probe assay has high sensitivity $(89.6 \%, 95 \% \mathrm{CI}$; $182.5-94.5 \%)$ and moderate specificity $(65.4 \%$, $95 \%$ CI; $44.3-82.7 \%$ ) in the detection of rifampicin. The kappa agreement between LPA and phenotypic drug susceptibility testing (PDST) was 0.53. The overall performance of LPA for the detections of rifampicin in this study was higher than previously reported by Scott et al. [10]. Scott and co reported 40\% (95\% CI 5.27 - $85.34 \%$ ) sensitivity of LPA to detect rifampicin in a study to compare the performance of 
GeneXpert with other nucleic acid assays. A sensitivity of $60.00 \%$ (95\% CI; $14.66-94.73 \%$ was reported in a study to evaluate genotype MTBDR plus assay as a tool for drug resistance survey [11]. In another study, Chryssanthou et al. [12] report a sensitivity of $75.00 \%$ (95\% CI; $19.41-99.37 \%)$. While Maschmann et al [13] reported $82.14 \%$ (95\% C. I; $63.11-93.94 \%$ ). The specificities reported by previous authors were higher than those reported in this study [11-13]. Also, Lower specificities (66.67\%, 95\% C.I; $9.43-99.5 \%$ and $50.00 \%$, $95 \%$ C. I; 1.20 $98.72 \%$ ) were reported by Sangsayunh et al [14] and Miolt et al $[15,16]$. The overall performance of LPA for the detection of INH in this study was moderate, and the sensitivity was $65.4 \%, 95 \% \mathrm{C}$. I; 44.3- 82.7\%. This result was higher than the previous finding by Rigouts et al. [11] (54.90\%), Maschmann Rde et al. [13] (60.42\%), Dorman et al. [17] $62.07 \%$ and Scott et al. [10] (66.67\%). However, the specificity of LPA for the detection of isoniazid resistance was lower in this study than the previously reported $[10,11,13,17]$.

The overall sensitivity of LPA to detect MDRTB in this study was higher than previous studies in Brazil, where the sensitivity was 59.2\%) [13]; $60.00 \%$ in Tanzania [11]. Our finding is similar to $69.64 \%$ in China [18]. The performance of LPA in this study was low compare to the most recent studies. Meaza et al. [19] reported higher sensitivity $(96.4 \%)$ to detect MDR-TB by LPA. He et al. [20], in another study that uses LPA version 1.0 reported lower performance for detecting rifampicin, isoniazid and multi-drug resistance TB. The low performance reported by $\mathrm{He}$ and co maybe because of the difference in DNA isolation used in the current LPA version and those used in the earlier version. It was also noted that LPA version 2.0 has a lower chance of cross-contamination than LPA version 1.0 [21].

The low performance of LPA version 2 in this study compared with the previous study uses the same versions maybe because of the participant's sputum status, study design, and technical knowhow.

Our finding revealed that the performance of LPA among HIV negative patients was better than among HIV positive patients. Also, the Kappa agreement between LPA and cultures for the detections of rifampicin among HIV negative patient was higher than in patients with HIV ( 0.57 vs 0.40$)$. The difference in the diagnostic performance of LPA among patient with HIV and patients without HIV in this study is perhaps because of the low bacillary load among HIV positive patients with $\mathrm{TB}$. It was observed that the limit of detection of MTB by LPA is 10,000 $\mathrm{CFU} / \mathrm{ml}$ while that of culture is $10-100 \mathrm{CFU} / \mathrm{ml}$. LPA likely missed specimens with low bacillary load [5, 22].

The sensitivity and specificity of LPA for detection of INH resistance were higher among patients without HIV than patient with HIV (77.4\% and $63.6 \%$ vs $73.9 \%$ and $50.0 \%$ ). Similar results were obtained for the detection of multidrug resistant tuberculosis.

\section{CONCLUSION}

Analyzing the overall performance of LPA in this study gave moderate sensitivities among patients with HIV and patients without HIV. This test's performance for detecting INH, RMP mono resistance, and MDR agreed with the gold standard. There is a need for comparing the performance of LPA against phenotypic DST and genomic sequencing in countries that started implementing the use of LPA in less than 3 to 5 years.

This study is limited by: the performance of LPA requires caution for interpretation and is dependent on the power of observation; there is also a need for interlaboratory competency assessment to assess the performance of workers at private and zonal reference laboratories. In addition, there was a high rate of contaminations which may affect the interpretation of the results presented in this study.

Funding: No funding sources.

Competing interest: None declared.

\section{Acknowledgement}

We are most grateful to the Zankli TB Research Laboratory's technical staff, Karu, Nigeria, for their kind support during this study.

\section{Ethical considerations}

The Health Research Ethics Committee of the Benue State University Teaching Hospital, Makurdi, Nigeria gave the permission (BSUTH/MKD/HREC2013B/2017/0011) for the conduct this study

\section{REFERENCES}

1. WHO. Global Tuberculosis Report 2018. Geneva; 2018. 
2. World Health Organization. Global tuberculosis report 2016. 2016.

3. Cadmus S, Palmer S, Okker M, Dale J, Gover K, Smith N, et al. Molecular analysis of human and bovine tubercle bacilli from a local setting in Nigeria. J Clin Microbiol. 2006; 44: 29-34.

4. Gupta-Wright A, Tomlinson GS, Rangaka MX, Fletcher HA. World TB Day 2018: The Challenge of Drug Resistant Tuberculosis. F1000Res. 2018 Feb 22;7:217.

5. Ejeh EF, Undiandeye A, Akinseye VO, Okon KO, Kazeem HM, Kudi CA, et al. Diagnostic performance of GeneXpert and Ziehl-Neelson microscopy in the detection of tuberculosis in Benue State, Nigeria. Alexandria J Med. 2018; 54:529-33.

6. Heidebrecht CL, Podewils LJ, Pym AS, Cohen $\mathrm{T}$, Mthiyane T, Wilson D. Assessing the utility of Xpert(®) MTB/RIF as a screening tool for patients admitted to medical wards in South Africa. Sci Rep. 2016 Jan 20;6:19391.

7. van Cleeff MRA, Kivihya-Ndugga LE, Meme H, Odhiambo JA, Klatser PR. The role and performance of chest X-ray for the diagnosis of tuberculosis: A cost-effective analysis in Nairobi, Kenya. BMC Infect Dis. 2005.

8. WHO. Chest Radigraphy in Tuberculosis Detection. 2016;:1-44. https://apps.who.int/iris/bitstream/handle/10665/ 252424/9789241511506-

eng.pdf;jsessionid=F5475BB16D969A2440AC6 8449481FE14? sequence $=1$.

9. Addo KK, Addo SO, Mensah GI, Mosi L, Bonsu FA. Genotyping and drug susceptibility testing of mycobacterial isolates from population-based tuberculosis prevalence survey in Ghana. BMC Infect Dis. 2017 Dec 2;17(1):743

10. Scott LE, McCarthy K, Gous N, Nduna M, Van Rie A, Sanne I, et al. Comparison of Xpert MTB/RIF with other nucleic acid technologies for diagnosing pulmonary tuberculosis in a high HIV prevalence setting: a prospective study. PLoS Med. 2011 Jul;8(7):e1001061.

11. Rigouts L, Hoza AS, De Rijk P, Torrea G, Chonde TM, Basra D, et al. Evaluation of the Genotype ${ }^{\circledR}$ MTBDRplus assay as a tool for drug resistance surveys. Int J Tuberc Lung Dis. 2011 Jul;15(7):959-65.

12. Chryssanthou E, Ängeby K. The GenoType $®$ MTBDRplus assay for detection of drug resistance in Mycobacterium tuberculosis in Sweden. APMIS. 2012;120:405-9.
13. Maschmann Rde A, Sá Spies F, Nunes Lde S, Ribeiro AW, Machado TR, Zaha A, Rossetti ML. Performance of the GenoType MTBDRplus assay directly on sputum specimens from Brazilian patients with tuberculosis treatment failure or relapse. J Clin Microbiol. 2013 May;51(5):1606-8.

14. Sangsayunh P, Chuchothawon C. Genotype MTBDR plus (Hain) test in suspected MDR-TB patients. J Med Assoc Thail. 2014;97:1028-32.

15. Miotto P, Saleri N, Dembelé M, Ouedraogo M, Badoum G, Pinsi G, et al. Molecular detection of rifampin and isoniazid resistance to guide chronic TB patient management in Burkina Faso. BMC Infect Dis. 2009; Aug 28;9:142.

16. Miotto P, Badoum G, Ouedraogo M, Bayala NR, Dembele SM, Saleri N, et al. Rapid detection of MDR-TB to guide patients' management: A feasibility study in burkina FASO. Infection. 2009; 37 (Suppl. II): 1-111.

17. Dorman SE, Chihot VN, Lewis JJ, Van Meulen M Der, Mathem B, Beylis N, et al. Genotype MTBDRplus for direct detection of Mycobacterium tuberculosis and drug resistance in strains from gold miners in South Africa. $J$ Clin Microbiol. 2012; 50:1189-94.

18. Chen C, Kong W, Zhu L, Zhou Y, Peng H, Shao $\mathrm{Y}$, et al. Evaluation of the GenoType ${ }^{\circledR}$ MTBDRplus line probe assay on sputumpositive samples in routine settings in China. Int J Tuberc Lung Dis. 2014; 18:1034-9.

19. Meaza A, Kebede A, Yaregal Z, Dagne Z, Moga $S$, Yenew B, et al. Evaluation of genotype MTBDRplus VER 2.0 line probe assay for the detection of MDR-TB in smear positive and negative sputum samples. BMC Infect Dis. 2017; Apr 17;17(1):280.

20. He Y, Han C, Chang KF, Wang MS, Huang TR. Diagnostic performance of GenoType MTBDRplus on culture specimens in smearnegative retreatment tuberculosis patients. Int $J$ Clin Exp Pathol. 2017;10:3528-53.

21. Singh BK, Sharma SK, Sharma R, Sreenivas V, Myneedu VP, Kohli M, et al. Diagnostic utility of a line probe assay for multi-drug resistant-TB in smear-negative pulmonary tuberculosis. PLoS One. $2017 ; 22 ; 12(8): \mathrm{e} 0182988$.

22. Ninan MM, Gowri M, Christopher DJ, Rupali P, Michael JS. The diagnostic utility of line probe assays for multidrug-resistant tuberculosis. Pathog Glob Health. 2016;110:194-9. 